\title{
RETRACTED ARTICLE: Investor sentiment, stock market valuation and merger activity
}

\author{
Benjamin R. Auer • Frank Schuhmacher
}

Received: 10 June 2011/Accepted: 4 November 2011/Published online: 4 December 2011 (C) Springer-Verlag 2011

The article 'Investor sentiment, stock market valuation and merger activity', published OnlineFirst in December 2011 in the International Review of Economics, has been retracted at the request of the authors. Due to missing references to important sources the authors decided to request the manuscript to be retracted in order to avoid a legal dispute. The Editor-in-Chief and the Publisher concurred with the decision. 